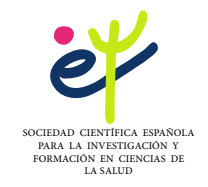

European Journal of Education and Psychology

2021, Vol. 14, No 1 (Págs. 1-18)

Eur. j. educ. psychol. e-ISSN 1989-2209 https://revistas.uautonoma.cl/index.php/ejep doi: $10.32457 /$ ejep.v14i1.1432

\title{
Miedo al Covid, agotamiento y cinismo: su efecto en la intención de abandono universitario
}

\section{Fear of Covid, exhaustion and cynicism: its effect on the intention to drop out of college}

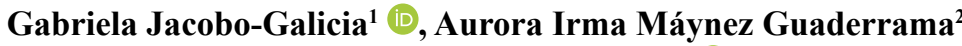 \\ \& Judith Cavazos-Arroyo ${ }^{3 *}$ (D) \\ ${ }^{1}$ Universidad Autónoma de Baja California, México; ${ }^{2}$ Universidad Autónoma de Ciudad Juárez, \\ México; ${ }^{3}$ Centro Interdisciplinario de Posgrados e Investigación Universidad Popular Autónoma del \\ Estado de Puebla, México.
}

\begin{abstract}
Resumen
La pandemia de Covid-19 ha impactado sin lugar a duda al sector educativo. Entre sus posibles consecuencias se encuentran la aparición y desarrollo del síndrome de Burnout y la intención de abandono por parte de estudiantes. Por ello, el objetivo de esta investigación fue determinar si —en estudiantes universitarios mexicanos- el miedo al Covid-19, el agotamiento emocional y el cinismo son antecedentes de la intención de abandono escolar. Se realizó una investigación cuantitativa, empírica, no experimental y transversal, en 478 estudiantes durante los meses de mayo y junio de 2020, en pleno periodo de pandemia de Covid-19. La técnica de análisis fue un modelo de ecuaciones estructurales con mínimos cuadrados parciales [PLS-SEM]. La evidencia indica que el miedo al Covid-19 impacta directa e indirectamente en la intención de abandono, a través del agotamiento emocional y del cinismo. Las limitaciones del estudio implican el uso de un muestreo no probabilístico. El valor de la investigación radica en la contribución a la comprensión de los efectos del miedo a contraer la enfermedad sobre la intención de abandonar los estudios universitarios. Se concluye que el miedo al Covid-19, el cinismo y el agotamiento influyen directa e indirectamente sobre la intención de abandono.
\end{abstract}

Palabras clave: Deserción escolar, Burnout, miedo. * Para Correspondencia: Judith Cavazos-Arroyo. Centro Interdisciplinario de Posgrados e Investigación,
Universidad Popular Autónoma del Estado de Puebla, México judith.cavazos@upaep.mx 


\begin{abstract}
The Covid-19 pandemic has undoubtedly affected the education sector; its possible consequences include the development of burnout and the intention to dropout. Thus, the objective of this research was to determine if, in Mexican university students, fear of Covid-19, emotional exhaustion and cynicism are antecedents of the intention to drop out of school. A quantitative, empirical, non-experimental and cross-sectional investigation was carried out on 478 students during the Covid-19 pandemic. The analysis technique was a model of Structural Equations with Partial Least Squares [PLS-SEM]. The evidence indicates that the fear of Covid-19 directly and indirectly affects the intention to dropout, through emotional exhaustion and cynicism. The limitations of the study imply the use of a non-probability sampling. The value of the research lies in the contribution to the understanding of the effects of the fear of contracting the disease on the intention to abandon university studies. It is concluded that the fear of Covid-19, cynicism and exhaustion directly and indirectly influence the intention to abandon school.
\end{abstract}

Keywords: College dropout, Burnout, fear.

\title{
INTRODUCCIÓN
}

A finales de 2019 se detectaron en Wuhan, China casos de neumonía atípica (CNN Español, 2020); posteriormente, se identificó un tipo de coronavirus de rápida propagación con efectos variados sobre la salud humana. Unos meses después, la Organización Mundial de la Salud [OMS] declaró que la humanidad enfrentaba una pandemia, ya que el virus se había propagado a más de 155 países, rebasando en poco tiempo 5 millones de personas infectadas y más de 300,000 fallecidas en el mundo (Johns Hopkins Coronavirus Resource Center, 2020). Los efectos en organizaciones de diferentes sectores fueron inmediatos: algunos vieron incrementada exponencialmente su demanda, y en el caso del sector educativo se realizaron ajustes y se adoptaron sistemas a distancia para continuar operando. La Organización de las Naciones Unidas para la Educación, la Ciencia y la Cultura [UNESCO] (2020) declaró que aproximadamente 138 países cerraron sus instituciones educativas, lo que generó consecuencias diversas en los estudiantes (Van Lancker \& Parolin, 2020).

En México, a principios de mayo del 2020, la Secretaría de Educación Pública [SEP] señaló que más de 5 millones de estudiantes del nivel superior, mantenían el receso escolar presencial asociado con la pandemia, pero continuaban con actividades a distancia (Wong, 2020). En el corto plazo, los desafíos principales incluían altas tasas de deserción o reducción sustancial de la matrícula universitaria (Vanguardia, 2020). La pandemia representa un llamado a la acción para investigadores psicosociales, ya que es de vital importancia 
comprender sus consecuencias (Asmundson \& Taylor, 2020). Estudiantes universitarios urbanos sufrieron más ansiedad y miedo aunados a las consecuencias económicas, sociales y académicas de la vida en aislamiento (Huang et al., 2020). En el caso de México, se identificó que los estudiantes se sentían estresados, angustiados e incluso deprimidos por la incertidumbre, la falta de contacto social cara a cara, el cierre de las instalaciones escolares y las medidas de distanciamiento social (Araujo Gómez, 2020). Por ello, el objetivo de este trabajo de investigación fue determinar si, en estudiantes universitarios mexicanos, el miedo al Covid-19, el agotamiento emocional y el cinismo son antecedentes de la intención de abandono escolar.

\section{Miedo al Covid-19 y agotamiento emocional}

Para comprender las repercusiones psicológicas y psiquiátricas de una pandemia, deben considerarse y observarse emociones como el miedo (Ornell et al., 2020). Esta emoción es uno de los aspectos psicológicos de la pandemia provocada por Covid-19 (Pakpour \& Griffiths, 2020). El miedo es un estado emocional displacentero, un mecanismo de defensa disparado por la percepción de estímulos potencialmente amenazantes que activa procesos biológicos de preparación (Ornell et al., 2020; Pakpour \& Griffiths, 2020). El miedo al contagio es un símbolo de respuesta a amenazas o desaprobación a contraer una enfermedad, en lugar del miedo en sí mismo (Heijnders \& Van Der Meij, 2006). Se caracteriza por respuestas afectivas como evitar a otros, tomar precauciones extremas y utilizar expresiones verbales asociadas al miedo (Meisenhelder \& La Charite, 1989). Investigaciones sobre el miedo al contagio del SIDA han encontrado que éste contribuye al aislamiento, al rechazo y a la discriminación hacia las personas contagiadas (Douglas et al., 1985).

Probablemente, el miedo al Covid-19 se debe a su novedad y a la incertidumbre de la magnitud del daño que causa (Asmundson \& Taylor, 2020). Con sus tasas de infección y mortalidad, el virus ha tenido un impacto psicológico universal que ha generado una plétora de manifestaciones psiquiátricas que se han reflejado en histeria y miedo masivos; además, los encierros nacionales pueden provocar a largo plazo pánico agudo, ansiedad, conductas compulsivas, paranoia, depresión y síndrome post-traumático (Dubey et al., 2020). Se ha encontrado que el aislamiento de individuos en combinación con el miedo al contagio, la cuarentena y el estigma, así como con la sobrecarga de información potencial, causan estrés crónico y una carga sobre la salud mental, planteando factores de riesgo para el agotamiento, la ansiedad y la depresión (Burtscher et al., 2020). Además del miedo al Covid-19, la pandemia ha influido en aspectos como: organización familiar, cierre de escuelas, empresas y lugares públicos, cambios laborales y aislamiento (Ornell et al., 2020). En el sector educativo, el aislamiento de los estudiantes durante la pandemia provocó niveles más altos de ansiedad, depresión y estrés (Odriozola-González et al., 2020). 
Aunque los estudiantes universitarios no son empleados, sus actividades están estructuradas de forma que se puede considerar un "trabajo" (Lin \& Huang, 2014). A semejanza de la fuerza laboral, los estudiantes están sujetos a condiciones estresoras tareas, fechas de entrega y largas horas de dedicación- que provocan agotamiento (Law, 2007). Este cúmulo de actividades y responsabilidades provoca estrés relacionado a la labor estudiantil, que a su vez puede llevar al agotamiento y derivar en Síndrome de Burnout Académico [SBA] (Ferrel et al., 2017). El agotamiento emocional es la dimensión más notable del Burnout, es su síntoma inicial (Maslach et al., 2001). En el entorno académico, se refiere a las sensaciones de fatiga, nerviosismo y frustración por las demandas escolares (Schaufeli et al., 2002). Debido al agotamiento, los alumnos dejan de entusiasmarse por sus estudios y no pueden concentrarse en ellos, ya que sus recursos emocionales se agotan (Schaufeli \& Bakker, 2004). Estudios realizados durante la pandemia de Covid-19 encontraron que ésta aumentó el estrés psicológico en estudiantes, con agotamiento emocional subsecuente (Gritsenko et al., 2020). Enfrentar amenazas como el Covid-19, somete a las personas a altos niveles de presión psicológica que, de acuerdo a experiencias en pandemias anteriores, se traduce en sentimientos de miedo y pánico, generando impactos psicológicos importantes (Tsamakis et al., 2020). Luego, es posible suponer que:

H1. El miedo al Covid-19 influye en el agotamiento emocional académico.

\section{Agotamiento emocional y cinismo en estudiantes}

El agotamiento en el contexto de educación superior se relaciona a sentimientos de tensión y estrés vinculados a la vida académica; se manifiesta a través de fatiga crónica por la carga académica y las demandas escolares (Salmela-Aro \& Read, 2017). El cinismo representa la dimensión cognitiva del Burnout, es una estrategia de afrontamiento ineficaz que se manifiesta como actitudes indiferentes, distantes y negativas hacia las actividades escolares (Lee et al., 2020); se exhibe como desapego hacia los estudios cuando los resultados -en términos de recompensas o consecuencias - no se asocian al esfuerzo; por lo tanto, se utiliza el distanciamiento cognitivo — generación de indiferencia o actitud cínica — cuando se está exhausto y no hay apoyo (Lee et al., 2020; Maslach et al., 2001).

El cinismo es una actitud de frustración y desilusión (Lee et al., 2020). Su origen es el desajuste percibido entre las expectativas y la realidad; por ejemplo, las expectativas de los estudiantes universitarios en su entorno (Wei et al., 2015) o bien, consigo mismos y sus bajos resultados de autoestima, esperanza y satisfacción con la vida (Dangel et al., 2018). Trabajos previos (Kim et al., 2015; Schaufeli et al., 2002) señalan que los alumnos agotados emocionalmente son más proclives a presentar cinismo y poca sensación de logro durante su vida académica. Por tanto, se propone que:

$\mathrm{H} 2$. El agotamiento emocional conduce al cinismo. 


\section{Miedo al Covid-19 e intención de abandono escolar}

Durante el brote de SARS se reportaron síntomas de ansiedad, aburrimiento, enojo, incertidumbre y miedo al contagio (Maunder et al., 2003). El miedo se vincula con aspectos como el desarrollo de hábitos, comportamientos y estigmas (Badahdah et al., 2020). Especialmente el miedo asociado a la seguridad personal y la carga psicológica en una pandemia, pueden tener consecuencias psicosociales como el ausentismo y el abandono de los estudios (Seale et al., 2009).

La pandemia de Covid-19 impactó al sector educativo en distintas formas; entre ellas, el aislamiento social y el abandono escolar (Nicola et al., 2020). La deserción escolar a nivel universitario es un problema global y comprender los motivos que llevan a los estudiantes a dejar prematuramente la escuela es un tema de investigación frecuente (Farr-Wharton et al., 2018). Entre otros factores, se asocia con la falta de satisfacción con los estudios, la calidad de la educación, la relación entre profesor y estudiante, el Burnout y la falta de soporte social (Farr-Wharton et al., 2018; Mostert \& Pienaar, 2020). Se afirma que la deserción escolar tiene consecuencias personales y sociales negativas, ya que implica menores ingresos personales, menor felicidad percibida, mayor depresión reportada y mayor estrés (Faas et al., 2018). La intención de abandonar los estudios se considera una señal de alerta temprana de la deserción escolar (Brandstätter $e t$ al., 2006). Por ello, comprender las variables que la afectan puede facilitar el diseńo de servicios de apoyo para evitar el abandono escolar (Schnettler et al., 2020). La intención de abandono debe considerarse un indicador de un patrón de apatía hacia los estudios, el cual, si se manifiesta a través de inasistencia a clases, postergación de trabajos o no presentación de exámenes, puede conducir a la deserción escolar (Moneta, 2011).

La comunidad universitaria puede tener miedo de contagiarse del virus. Es posible que los alumnos, buscando su protección, eviten asistir a las instalaciones escolares; incluso, pueden abandonar temporal o definitivamente sus estudios. Trabajos recientes indican que las preocupaciones de salud y la ansiedad en las pandemias pueden derivar en comportamientos preventivos desfavorables (Jungmann \& Witthöft, 2020); es probable que las personas amenazadas por el coronavirus tengan comportamientos de autoprotección (Jørgensen et al., 2020). Por ello, se postula que:

H3. El miedo al Covid-19 influye en la intención de abandono escolar.

\section{Agotamiento emocional e intención de abandono escolar}

La intención de dejar la escuela se basa en sentimientos de incompetencia, falta de involucramiento personal e indiferencia a ser educado; en términos generales, representa los sentimientos de los estudiantes respecto a su educación (Frostad et al., 2015). La retención o prevención de la intención de abandono escolar es un problema complejo que enfrentan los estudiantes, los administradores escolares y los docentes (Jüttler, 2020). Desde la perspectiva de la gestión, el abandono escolar lleva a pérdidas financieras por la disminución de ingresos (Law, 2007). 
Los vínculos entre los componentes del Burnout son similares entre trabajadores y estudiantes; por ello, es probable que sus patrones de Burnout sean similares (Moneta, 2011). En el entorno laboral, el agotamiento emocional conduce a la intención de abandono (Hwang et al., 2014; McKnight et al., 2009). En el ámbito escolar, puede llevar a la ausencia de clases, pérdida de interés, bajo rendimiento académico y la decisión de abandono de los estudios (Law, 2007). Por ello, se propone que:

$\mathrm{H} 4$. El agotamiento emocional conduce a la intención de abandono escolar.

\section{Cinismo e intención de abandono escolar}

El cinismo académico es un fenómeno global cada vez más común entre estudiantes universitarios, aunque sus efectos se han subestimado (Kachel et al., 2020; Wei et al., 2015). El cinismo implica una actitud indiferente que crea un distanciamiento cognitivo ante una situación que excede las capacidades (Maslach et al., 2001). En la educación superior se manifiesta como una actitud indiferente o distante hacia el estudio, una pérdida de interés en el trabajo académico y la percepción de que la educación no es significativa (Salmela-Aro $\&$ Read, 2017). Una actitud escolar cínica implica una creencia negativa por la percepción de disparidad entre las expectativas y las experiencias universitarias (Wei et al., 2015).

En la empresa se ha encontrado que el cinismo predice la intención de abandono (Çaylak \& Altuntaş, 2017; Jyoti \& Rani, 2019; Lingard, 2003). En la escuela, es un factor que explica el abandono de los estudios (Bask \& Salmela-Aro, 2013). El cinismo escolar puede generar resultados desfavorables como un menor sentido de bienestar e incluso la decisión de abandonar la escuela (Wei et al., 2015). Luego, el cinismo desplegado como una actitud indiferente y pérdida de interés en el estudio, puede llevar a los estudiantes a considerar que no es importante atender las tareas escolares; esa falta de significado conduce luego al abandono de los estudios (Lee et al., 2020). Por ello, se postula que:

$\mathrm{H} 5$. El cinismo predice la intención de abandono escolar.

\section{MÉTODO}

Participantes. En total participaron 478 estudiantes. El $54.4 \%$ de ellos de sexo masculino y $45.6 \%$ de sexo femenino. El 72.6\% tenían entre 19 y 22 ańos de edad; $27.2 \%$ cursaba el cuarto semestre de licenciatura, el $14 \%$ tercero, $12.6 \%$ sexto y $11.1 \%$ quinto. $61.3 \%$ manifestó no trabajar y $38.7 \%$ realiza actividades laborales. El $90 \%$ vive con padres o familiares, $92.7 \%$ eran solteros(as). El 96.2\% estudiaba en una universidad pública.

Instrumentos. En una primera fase, se revisó la literatura para identificar antecedentes sobre las variables estudiadas. El agotamiento y el cinismo se valoraron con ítems de las escalas de Schaufeli, Martínez, Pinto, Salanova y Bakker (2002) y de Hederich-Martínez y 
Caballero-Domínguez (2016). La intención de abandono escolar con ítems de Frostad, Pijl, y Mjaavatn (2015). El miedo al Covid-19, con ítems adaptados de Snell Jr. y Finney (1998) (ver Tabla 1). Las escalas se adaptaron al contexto; su medición se realizó con ítems Likert desde $1=$ "nunca" hasta 5="muy frecuentemente".

Tabla 1.

Escalas utilizadas

\begin{tabular}{l}
\hline \multicolumn{1}{c}{ Agotamiento } \\
Ag1.Me siento \\
emocionalmente agotado \\
por mis estudios. \\
Ag2. Cuando termino mi \\
día me siento físicamente \\
agotado. \\
Ag3.Estoy cansado en \\
la mańana cuando me \\
levanto y tengo que \\
afrontar otro día en la \\
universidad. \\
Ag4. Estudiar o ir a clases \\
todo el día me hace sentir \\
tenso.
\end{tabular}

Miedo al Covid-19

Cv1. Me siento ansioso por la epidemia de Covid-19.

Cv2. Tengo miedo de contagiarme de Covid-19.

Cv3. Me asusta contagiarme de Covid-19 por el contacto con

un familiar, amigo, vecino o alguien en la calle.

Cv4. Me siento ansioso cuando hablo con familiares, amigos o vecinos sobre el Covid-19.

Cv5. El Covid-19 es una experiencia muy estresante para mi.

Cv6. El Covid-19 ha comenzado a afectar mis relaciones personales.

Cv7. La propagación del Covid-19 me estresa.

Cv8. Me preocupa que yo pueda tener el virus Covid-19.

Cv9. Tengo miedo de contagiar el Covid-19 a un amigo,

familiar o vecino.

Ag5. Estoy exhausto de

tanto estudiar.

\section{Cinismo}

Intención de abandonar los estudios

Ci1. Desde que me inscribí Ia1. Con frecuencia pienso en dejar la escuela y en lugar de en la universidad he ido estudiar, buscar un trabajo.

perdiendo interés por mis Ia2. No entiendo qué es lo que estoy haciendo en la escuela. estudios.

Ci2. Cada vez tengo Ia3. Con frecuencia pienso en dejar esta escuela porque las menos entusiasmo por estudiar. materias son muy teóricas.

Ia4. Realmente siento que estoy perdiendo el tiempo en la escuela.

Ci3. Pienso que mis Ia5. Con frecuencia pienso en abandonar la escuela.

estudios no son realmente útiles.

Ia6. Nunca voy a tener éxito en la escuela, incluso si pongo todo mi empeño.

Ci4. Dudo de la Ia7. Con frecuencia pienso en dejar la escuela debido a los importancia y valor de mis continuos conflictos que tengo con mis maestros. estudios.

Ci5. Dudo del impacto que pueda tener lo que Ia8. Con frecuencia pienso en abandonar la escuela debido a problemas con mi familia. estudio.

Fuente: Elaboración propia. 
Procedimiento. Se realizó una investigación cuasiexperimental, cuantitativa, empírica, no probabilística y transversal. Durante los meses de mayo y junio del 2020, se aplicó una encuesta electrónica habilitada en e-encuesta, que se difundió a través de correos electrónicos de profesores, redes sociales institucionales de las universidades y plataformas institucionales de educación a distancia. Se utilizó una muestra no probabilística, por conveniencia, sujeta a la disposición de los estudiantes a participar. Se garantizó el anonimato a los participantes y no se les solicitó información personal.

Análisis de datos. Se utilizó la técnica de ecuaciones estructurales con mínimos cuadrados parciales (PLS-SEM) para el análisis, por su utilidad para explorar y predecir, probar y validar modelos exploratorios (Henseler et al., 2009). En el análisis estadístico inferencial de los datos se siguieron dos etapas: en la primera, se revisaron los atributos psicométricos del modelo de medición (validez de convergencia y discriminante); en la segunda, se valoró el modelo estructural. El software estadístico fue Smart PLS 3.2.

La validez convergente se estableció con base en las cargas factoriales (valor y significancia estadística), el indicador alfa de Cronbach, la fiabilidad compuesta y la varianza extraída media (ver tabla 2). Las cargas factoriales de los ítems mostraron valores entre 0.627 (Cv1) y 0.808 (Ia5), con valores t mayores a 1.96. El alfa de Cronbach y la fiabilidad compuesta de los constructos excedieron el punto de corte mínimo de 0.70 (Bagozzi \& Yi, 1988; Fornell, C., \& Larcker, 1981; Seidel \& Back, 2009). En lo referente a la varianza extraída media, tres de los constructos mostraron valores superiores a 0.50 (Martínez Ávila \& Fierro Moreno, 2018); solo el agotamiento tuvo un valor marginal a ese punto de corte (0.492). Luego, puede afirmarse que el modelo de medición tiene validez de convergencia.

Tabla 2.

Validez convergente

\begin{tabular}{|c|c|c|c|c|c|c|}
\hline \multirow[b]{2}{*}{ Variable } & \multicolumn{3}{|c|}{ Carga factorial } & \multirow[t]{2}{*}{$\begin{array}{c}\text { Alfa de } \\
\text { Cronbach } \\
\end{array}$} & \multirow[t]{2}{*}{$\begin{array}{l}\text { Fiabilidad } \\
\text { compuesta }\end{array}$} & \multirow[t]{2}{*}{$\begin{array}{c}\text { Varianza extraída } \\
\text { media (AVE) }\end{array}$} \\
\hline & Ítem & Valor & Valor $\mathbf{t}$ & & & \\
\hline \multirow{5}{*}{ Agotamiento } & $\operatorname{Ag} 1$ & 0.747 & 32.669 & \multirow{5}{*}{0.743} & \multirow{5}{*}{0.829} & \multirow{5}{*}{0.492} \\
\hline & $\mathrm{Ag} 2$ & 0.674 & 20.411 & & & \\
\hline & $\mathrm{Ag} 3$ & 0.681 & 20.325 & & & \\
\hline & $\mathrm{Ag} 4$ & 0.692 & 19.416 & & & \\
\hline & $\mathrm{Ag} 5$ & 0.711 & 23.174 & & & \\
\hline \multirow{5}{*}{ Cinismo } & Ci1 & 0.743 & 27.765 & \multirow{5}{*}{0.821} & \multirow{5}{*}{0.875} & \multirow{5}{*}{0.583} \\
\hline & $\mathrm{Ci} 2$ & 0.767 & 30.288 & & & \\
\hline & $\mathrm{Ci} 3$ & 0.741 & 22.156 & & & \\
\hline & $\mathrm{Ci} 4$ & 0.795 & 36.299 & & & \\
\hline & $\mathrm{Ci} 5$ & 0.770 & 31.691 & & & \\
\hline
\end{tabular}




\begin{tabular}{|c|c|c|c|c|c|c|}
\hline \multirow[b]{2}{*}{ Variable } & \multicolumn{3}{|c|}{ Carga factorial } & \multirow[t]{2}{*}{$\begin{array}{c}\text { Alfa de } \\
\text { Cronbach }\end{array}$} & \multirow[t]{2}{*}{$\begin{array}{l}\text { Fiabilidad } \\
\text { compuesta }\end{array}$} & \multirow[t]{2}{*}{$\begin{array}{c}\text { Varianza extraída } \\
\text { media (AVE) }\end{array}$} \\
\hline & Ítem & Valor & Valor $\mathbf{t}$ & & & \\
\hline \multirow{8}{*}{$\begin{array}{l}\text { Intención de } \\
\text { abandono }\end{array}$} & Ia1 & 0.763 & 33.686 & \multirow{8}{*}{0.885} & \multirow{8}{*}{0.908} & \multirow{8}{*}{0.554} \\
\hline & $\mathrm{Ia} 2$ & 0.763 & 29.496 & & & \\
\hline & Ia3 & 0.761 & 32.298 & & & \\
\hline & $\mathrm{Ia} 4$ & 0.775 & 32.007 & & & \\
\hline & Ia5 & 0.808 & 36.113 & & & \\
\hline & $\mathrm{Ia} 6$ & 0.680 & 19.287 & & & \\
\hline & Ia7 & 0.702 & 20.889 & & & \\
\hline & Ia8 & 0.692 & 18.453 & & & \\
\hline \multirow{9}{*}{$\begin{array}{l}\text { Miedo al } \\
\text { COVID }\end{array}$} & Cv1 & 0.627 & 14.245 & \multirow{9}{*}{0.880} & \multirow{9}{*}{0.902} & \multirow{9}{*}{0.508} \\
\hline & Cv2 & 0.725 & 17.935 & & & \\
\hline & Cv3 & 0.698 & 15.962 & & & \\
\hline & Cv4 & 0.736 & 24.591 & & & \\
\hline & Cv5 & 0.770 & 31.461 & & & \\
\hline & Cv6 & 0.673 & 17.913 & & & \\
\hline & Cv7 & 0.802 & 38.004 & & & \\
\hline & Cv8 & 0.727 & 21.752 & & & \\
\hline & Cv9 & 0.638 & 15.019 & & & \\
\hline
\end{tabular}

Fuente: Elaboración propia.

La validez discriminante se estableció con el Heterotrait Monotrait Ratio (HTMT). En este caso, las correlaciones entre los indicadores del mismo constructo (monotraitheteromethod) deben exceder las correlaciones de los indicadores de diferentes constructos (heterotrait-heteromethod), el punto de corte máximo fue 0.85 (Henseler et al., 2015). Los resultados indican que existe validez discriminante (Tabla 3).

Tabla 3.

Validez discriminante

\begin{tabular}{lccc}
\hline & Agotamiento & Cinismo & Intención de abandono \\
\hline Agotamiento & & & \\
Cinismo & 0.566 & & \\
Intención de abandono & 0.480 & 0.742 & 0.203 \\
Miedo al COVID19 & 0.303 & 0.150 & \\
\hline
\end{tabular}

Fuente: Elaboración propia 


\section{RESULTADOS}

En la figura 1, se exhibe el modelo contrastado, con los coeficientes path de las relaciones estructurales y el valor $\mathrm{R}^{2}$ del agotamiento, del cinismo y de la intención de abandono. La calidad de predicción del modelo se determinó con base a los coeficientes path y al valor $\mathrm{R}^{2}$. En la evaluación de los path, se revisó la magnitud y significancia estadística. Los valores de los path estructurales coinciden con antecedentes teóricos y son significativos.

El coeficiente de determinación $\mathrm{R}^{2}$ muestra el grado en que, en el modelo estructural, una variable latente es explicada por otros constructos (do Nascimento \& da Silva Macedo, 2016). El criterio es: 0.75 sustancial, 0.50 moderado y 0.25 débil (Hair et al., 2016). En este modelo los resultados fueron: agotamiento [0.071], cinismo [0.202], intención de abandono [0.433]. Así, se explica de forma débil al agotamiento y al cinismo, y moderadamente la intención de abandono.

Figura 1.

Modelo contrastado

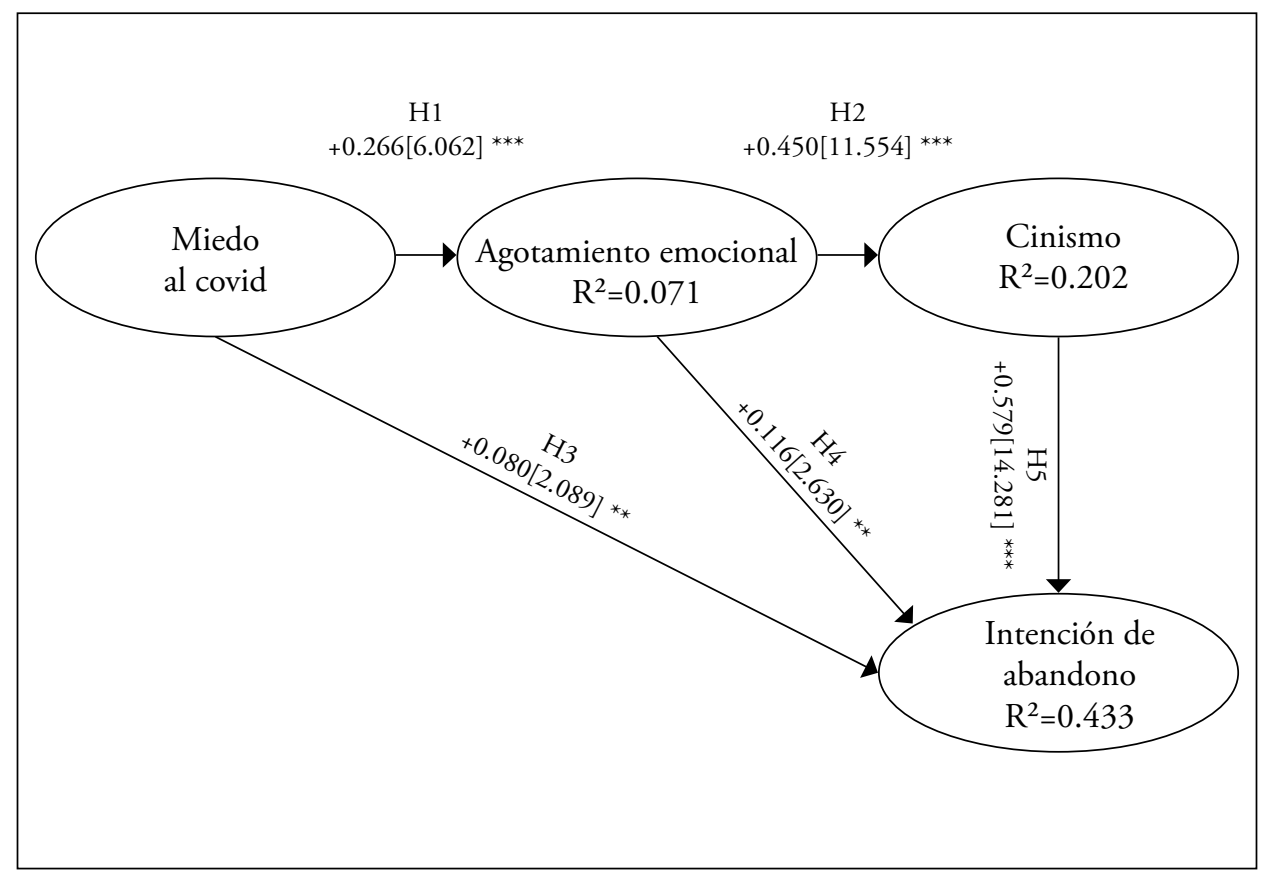

Fuente: Elaboración propia. 


\section{DISCUSIÓN}

Esta investigación analizó, en estudiantes universitarios mexicanos, si el miedo al Covid-19 provocó afectaciones de agotamiento emocional, cinismo e intención de abandono escolar. El miedo al Covid-19 se refleja en mayor medida en el estrés asociado a la propagación del virus, la preocupación de portarlo y el miedo de contagiarse. Estos resultados corroboran antecedentes que indican que las pandemias generan presiones que se traducen en sentimientos de miedo e impactos psicológicos (Tsamakis et al., 2020).

Esta investigación encontró que el miedo a Covid-19 provoca agotamiento. En el contexto estudiado, el agotamiento se despliega a través del cansancio emocional, el sentimiento de estar exhaustos y la tensión por estudiar. Estos hallazgos reiteran lo propuestos en trabajos previos que indican que el agotamiento disminuye el entusiasmo por los estudios, incrementa el estrés y genera agotamiento emocional (Gritsenko et al., 2020; Schaufeli \& Bakker, 2004). Asimismo, se identificó que el agotamiento deriva en cinismo, lo que coincide con antecedentes literarios (Kim et al., 2015; Schaufeli et al., 2002) que indican que los alumnos con agotamiento emocional tienen mayor proclividad a desarrollar cinismo y a experimentar baja sensación de logro académico. En esta investigación, el cinismo se refleja en las dudas de los alumnos sobre la importancia e impacto futuro de su formación y en la disminución de su entusiasmo por estudiar, lo que refleja una estrategia ineficaz ante la pandemia.

Se postuló que el miedo al Covid-19, el agotamiento y el cinismo llevan a la intención de abandono escolar. Los resultados fueron significativos para los tres casos. Por un lado, estos hallazgos confirman lo propuesto en trabajos previos que indican que el miedo genera intención de abandono (Jørgensen et al., 2020; Jungmann \& Witthöft, 2020), ya que en las pandemias surgen comportamientos de autoprotección, ansiedad y preocupaciones que pueden conducir al abandono temporal o definitivo de los estudios. Por otra parte, el agotamiento emocional influye en la intención de dejar los estudios. Nuestros resultados coinciden con antecedentes académicos que indican que, en el ámbito escolar, el agotamiento puede provocar que los alumnos se ausenten de clases, pierdan interés, tengan bajo rendimiento académico y finalmente decidan abandonar la escuela (Law, 2007). Finalmente, los resultados de este trabajo corroboran que el cinismo predice el abandono de los estudios (Bask \& Salmela-Aro, 2013; Wei et al., 2015), a consecuencia de la disminución del sentido de bienestar que genera indiferencia y pérdida de interés y significado de las actividades escolares (Lee et al., 2020). En este trabajo, la intención de abandono escolar se exhibe en pensamientos asociados con abandonar la escuela, el sentimiento de pérdida de tiempo al permanecer en ella, la consideración de dejarla y buscar un trabajo, y la falta de comprensión sobre sus actividades escolares. 


\section{CONCLUSIÓN}

Los resultados de este trabajo de investigación evidencian que durante la pandemia de Covid-19, el miedo al Covid-19, el agotamiento emocional y el cinismo conducen a la intención de abandono escolar. El impacto mayor lo recibe del cinismo, seguido del agotamiento y finalmente del miedo. Estos hallazgos tienen implicaciones tanto teóricas como prácticas. En este trabajo, el cinismo es la variable que predice en mayor medida la intención de abandono escolar. Es posible que durante la pandemia el cinismo de los estudiantes se haya incrementado. Las condiciones pandémicas pueden haber incrementado los cuestionamientos sobre la utilidad de los estudios. Si el estudiante duda del valor de sus estudios, es altamente probable que deje la escuela. Esta condición debe ser tomada en cuenta por las autoridades académicas a fin de que implementen medidas o programas que contrarresten este comportamiento. Es decir, se requiere diseñar estrategias de afrontamiento ante la situación.

Además, se identificó que el agotamiento provoca cinismo e intención de abandono. En ese sentido, es conveniente que los profesores sean conscientes de que se está enfrentando una situación atípica, los alumnos pueden no estar en condiciones de llevar a cabo sus actividades como normalmente lo hacen y, por tanto, se requiere de estrategias distintas a las usualmente utilizadas. La deserción escolar es un riesgo y los hallazgos pueden tener implicaciones que van más allá de las académicas. El abandono escolar asociado a la pandemia puede provocar reducción significativa de aprendizajes, desempleo, requerimientos de ayuda social, uso de sustancias tóxicas y crimen (Banco Mundial, 2020; Freeney \& O’Connell, 2009).

$\mathrm{Al}$ igual que otras investigaciones, existen limitaciones. Se utilizó una muestra no probabilística y las encuestas fueron recolectadas electrónicamente, por el confinamiento. Se recomienda que estudios futuros indaguen en las relaciones propuestas durante otras etapas del confinamiento y una vez que los estudiantes regresen físicamente a las instalaciones. Respecto a la intención de abandono, se sugiere profundizar en otros antecedentes. Consideramos importante indagar si la educación en línea tiene implicaciones en términos de la intención de abandono académico; lo anterior, ya que los estudiantes se encuentran en condiciones inusuales por un periodo prolongado y muchos de ellos se han enfrentado a restricciones tecnológicas como el acceso a Internet o la disponibilidad de equipo informático. Finalmente, para incrementar la validez externa, se llama a replicar este trabajo en otros contextos universitarios.

\section{REFERENCIAS}

Araujo Gómez, R. M. (2020, April 16). ¿Los universitarios están aislados o conectados? Expansión. https://politica.expansion.mx/voces/2020/04/16/coronavirus-coviduniversitarios-aislados-o-conectados 
Asmundson, G. J. G., \& Taylor, S. (2020). Coronaphobia: Fear and the 2019-nCoV outbreak. Journal of Anxiety Disorders, 70(January), 102196.

https://doi.org/10.1016/j.janxdis.2020.102196

Badahdah, A. M., Khamis, F., \& Mahyijari, N. Al. (2020). The psychological well-being of physicians during COVID-19 outbreak in Oman. Psychiatry Research, 289, 113053. https://doi.org/10.1016/j.psychres.2020.113053

Bagozzi, R. P., \& Yi, Y. (1988). On the evaluation of structural equation models. Journal of the Academy of Marketing Science, 16(1), 74-94. https://doi.org/10.1007/ BF02723327

Banco Mundial. (2020). COVID-19: Impacto en la Educación y respuestas de politica pública. https://openknowledge.worldbank.org/handle/10986/33696

Bask, M., \& Salmela-Aro, K. (2013). Burned out to drop out: Exploring the relationship between school burnout and school dropout. European Journal of Psychology of Education, 28(2), 511-528. https://doi.org/10.1007/s10212-012-0126-5

Brandstätter, H., Grillich, L., \& Farthofer, A. (2006). Prognose des studienabbruchs. Zeitschrift Fur Entwicklungspsychologie Und Padagogische Psychologie, 38(3), 121131. https://doi.org/10.1026/0049-8637.38.3.121

Burtscher, J., Burtscher, M., \& Millet, G. P. (2020). (Indoor) isolation, stress, and physical inactivity: Vicious circles accelerated by COVID-19? Scandinavian Journal of Medicine and Science in Sports, 30(8), 1544-1545. https://doi.org/10.1111/ sms.13706

Çaylak, E., \& Altuntaş, S. (2017). Organizational silence among nurses: The impact on organizational cynicism and intention to leave work. Journal of Nursing Research, 25(2), 90-98. https://doi.org/10.1097/JNR.0000000000000139

CNN Español. (2020, February 20). Cronología del coronavirus: así comenzó y se extendió el virus que tiene en alerta al mundo. CNN Español. https://cnnespanol.cnn. com/2020/02/20/cronologia-del-coronavirus-asi-comenzo-y-se-extendio-el-virusque-pone-en-alerta-al-mundo/

Dangel, T. J., Webb, J. R., \& Hirsch, J. K. (2018). Forgiveness and Suicidal Behavior: Cynicism and Psychache as Serial Mediators. The Journal of Psychology, 152(2), 7795. https://doi.org/10.1080/00223980.2017.1408555

do Nascimento, J. C. H. B., \& da Silva Macedo, M. A. (2016). Structural Equation Models using Partial Least Squares: An Example of the Application of SmartPLS ${ }^{\odot}$ in Accounting Research. Revista de Educação e Pesquisa Em Contabilidade, 10(3), 282-305. 
Douglas, C. J., Kalman, C. M., \& Kalman, T. P. (1985). Homophobia among physicians and nurses: An empirical study. Psychiatric Services, 36(12), 1309-1311. https://doi. org/10.1176/ps.36.12.1309

Dubey, S., Biswas, P., Ghosh, R., Chatterjee, S., Dubey, M. J., Chatterjee, S., Lahiri, D., \& Lavie, C. J. (2020). Psychosocial impact of COVID-19. Diabetes and Metabolic Syndrome: Clinical Research and Reviews, 14(5), 779-788. https://doi.org/10.1016/j. dsx.2020.05.035

Faas, C., Benson, M. J., Kaestle, C. E., \& Savla, J. (2018). Socioeconomic success and mental health profiles of young adults who drop out of college. Journal of Youth Studies, 21(5), 669-686. https://doi.org/10.1080/13676261.2017.1406598

Farr-Wharton, B., Charles, M. B., Keast, R., Woolcott, G., \& Chamberlain, D. (2018). Why lecturers still matter: the impact of lecturer-student exchange on student engagement and intention to leave university prematurely. Higher Education, 75(1), 167-185. https://doi.org/10.1007/s10734-017-0190-5

Ferrel, F., Ferrel, L., Cantillo, A., Jaramillo, J., \& Jimenez, S. (2017). Variables académicas y sociodemográficas relacionadas con el Síndrome de Burnout, en estudiantes de Ingenierías y Ciencias de la Salud de una universidad estatal de Colombia * Academic and demographic variables related to Burnout syndrome in engineering. Psicogente, 20 (38), 336-352.

Fornell, C., \& Larcker, D. F. (1981). Evaluating Structural Equation Models with Unobservable Variables and Measurement Error. Journal of Marketing Research, 18(1), 39-50.

Freeney, Y., \& O'Connell, M. (2009). Psychological, Economic and Academic Predictors of the Intention to Leave Early among a Sample of Irish Students. Combat Poverty Agency.

Frostad, P., Pijl, S. J., \& Mjaavatn, P. E. (2015). Losing All Interest in School: Social Participation as a Predictor of the Intention to Leave Upper Secondary School Early. Scandinavian Journal of Educational Research, 59(1), 110-122. https://doi.org/10.1 080/00313831.2014.904420

Gritsenko, V., Skugarevsky, O., Konstantinov, V., Khamenka, N., Marinova, T., Reznik, A., \& Isralowitz, R. (2020). COVID 19 Fear, Stress, Anxiety, and Substance Use Among Russian and Belarusian University Students. International Journal of Mental Health and Addiction, 1-7. https://doi.org/10.1007/s11469-020-00330-z

Hair, J. F., Hult, G. T. M., Ringle, C., \& Sarstedt, M. (2016). A Primer on Partial Least Squares Structural Equation Modeling (PLS-SEM) (2nd ed.). Los Angeles: SAGE.

Hederich-Martínez, C., \& Caballero-Domínguez, C. C. (2016). Validación del cuestionario Maslach Burnout Inventory-Student Survey (MBI-SS) en contexto académico colombiano. CES Psicología, 9(1), 1-15. https://doi.org/10.21615/cesp.9.1.1 
Heijnders, M., \& Van Der Meij, S. (2006). The fight against stigma: an overview of stigmareduction strategies and interventions. Psychology, Health \& Medicine, 11(3), 353363. https://doi.org/10.1080/13548500600595327

Henseler, J., Ringle, C. M., \& Sarstedt, M. (2015). A new criterion for assessing discriminant validity in variance-based structural equation modeling. Journal of the Academy of Marketing Science, 43(1), 115-135. https://doi.org/10.1007/s11747-014-0403-8

Henseler, J., Ringle, C. M., \& Sinkovics, R. (2009). The use of partial least squares path modeling in international marketing. In J. Henseler, C. M. Ringle, \& R. Sinkovics (Eds.), New Challenges to International Marketing (Advances in International Marketing) (pp. 277-319). Bingley: Emerald Group Publishing Limited.

Huang, L., Xu, F., \& Liu, H. (2020). Emotional responses and coping strategies of nurses and nursing college students during COVID-19 outbreak. MedRxiv. https://doi. org/10.1371/journal.pone.0237303

Hwang, J., Lee, J. J., Park, S., Chang, H., \& Kim, S. S. (2014). The impact of occupational stress on employee's turnover intention in the luxury hotel segment. International Journal of Hospitality \& Tourism Administration, 15(1), 60-77. https://doi.org/10.1 080/15256480.2014.872898

Johns Hopkins Coronavirus Resource Center. (2020). COVID-19 Dashboard by the Center for Systems Science and Engineering (CSSE).

Jørgensen, F. J., Bor, A., \& Petersen, M. B. (2020). Compliance Without Fear: IndividualLevel Predictors of Protective Behavior During the First Wave of the COVID-19 Pandemic. PsyArXiv. https://doi.org/10.31234/osf.io/uzwgf

Jungmann, S. M., \& Witthöft, M. (2020). Health anxiety, cyberchondria, and coping in the current COVID-19 pandemic: Which factors are related to coronavirus anxiety? Journal of Anxiety Disorders, 73, 102239. https://doi.org/10.1016/j. janxdis.2020.102239

Jüttler, M. (2020). Predicting economics student retention in higher education: The effects of students' economic competencies at the end of upper secondary school on their intention to leave their studies in economics. PLoS ONE, 15(2), 1-27. https://doi. org/10.1371/journal.pone.0228505

Jyoti, J., \& Rani, A. (2019). Role of burnout and mentoring between high performance work system and intention to leave: Moderated mediation model. Journal of Business Research, 98, 166-176. https://doi.org/10.1016/j.jbusres.2018.12.068

Kachel, T., Huber, A., Strecker, C., Höge, T., \& Höfer, S. (2020). Development of Cynicism in Medical Students: Exploring the Role of Signature Character Strengths and WellBeing. Frontiers in Psychology, 11, 1-16. https://doi.org/10.3389/fpsyg.2020.00328 
Kim, B., Lee, M., Kim, K., Choi, H., \& Lee, S. M. (2015). Longitudinal analysis of academic burnout in Korean middle school students. Stress and Health, 31(4), 281289. https://doi.org/10.1002/smi.2553

Law, D. W. (2007). Exhaustion in University Students and the Effect of Coursework Involvement. Journal of American College Health, 55(4), 239-245. https://doi. org/10.3200/JACH.55.4.239-245

Lee, M., Lee, K. J., Lee, S. M., \& Cho, S. (2020). From emotional exhaustion to cynicism in academic burnout among Korean high school students: Focusing on the mediation effects of hatred of academic work. Stress and Health, 376-383. https:// doi.org/10.1002/smi.2936

Lin, S. H., \& Huang, Y. C. (2014). Life stress and academic burnout. Active Learning in Higher Education, 15(1), 77-90. https://doi.org/10.1177/1469787413514651

Lingard, H. (2003). The impact of individual and job characteristics on "burnout" among civil engineers in Australia and the implications for employee turnover. Construction Management \& Economics, 21(1), 69-80. https://doi. org/10.1080/0144619032000065126

Martínez Ávila, M., \& Fierro Moreno, E. (2018). Aplicación de la técnica PLS-SEM en la gestión del conocimiento: un enfoque técnico práctico. RIDE Revista Iberoamericana Para La Investigación y El Desarrollo Educativo, 8(16), 130-164. https://doi. org/10.23913/ride.v8i16.336

Maslach, C., Schaufeli, W. B., \& Leiter, M. P. (2001). Job Burnout. Annual Review of Psychology, 52(1), 397-422. https://doi.org/10.1146/annurev.psych.52.1.397

Maunder, R., Hunter, J., Vincent, L., Bennett, J., Peladeau, N., Leszcz, M., Sadavoy, J., Verhaeghe, L. M., Steinberg, R., \& Mazzulli, T. (2003). The immediate psychological and occupational impact of the 2003 SARS outbreak in a teaching hospital. Cmaj, 168(10), 1245-1251.

McKnight, D. H., Phillips, B., \& Hardgrave, B. C. (2009). Which reduces IT turnover intention the most: Workplace characteristics or job characteristics? Information and Management, 46(3), 167-174. https://doi.org/10.1016/j.im.2009.01.002

Meisenhelder, J. B., \& LaCharite, C. (1989). Fear of contagion: The public response to AIDS. Image: The Journal of Nursing Scholarship, 27(1), 7-9.

Moneta, G. B. (2011). Need for achievement, burnout, and intention to leave: Testing an occupational model in educational settings. Personality and Individual Differences, 50(2), 274-278. https://doi.org/10.1016/j.paid.2010.10.002

Mostert, K., \& Pienaar, J. (2020). The moderating effect of social support on the relationship between burnout, intention to drop out, and satisfaction with studies of first-year 
university students. Journal of Psychology in Africa, 30(3), 197-202. https://doi.org/ 10.1080/14330237.2020.1767928

Nicola, M., Alsafi, Z., Sohrabi, C., Kerwan, A., Al-Jabir, A., Iosifidis, C., Agha, M., \& Agha, R. (2020). The socio-economic implications of the coronavirus pandemic (COVID-19): A review. International Journal of Surgery, 78, 185-193. https://doi. org/10.1016/j.ijsu.2020.04.018

Odriozola-González, P., Planchuelo-Gómez, Á., Irurtia, M. J., \& de Luis-García, R. (2020). Psychological effects of the COVID-19 outbreak and lockdown among students and workers of a Spanish university. Psychiatry Research, 290 (May), 113108. https://doi. org/10.1016/j.psychres.2020.113108

Organización de las Naciones Unidas para la Educación, la C. y la C. (2020). COVID-19 Educational Disruption and Response. https://en.unesco.org/covid19/ educationresponse

Ornell, F., Schuch, J. B., Sordi, A. O., \& Kessler, F. H. P. (2020). "'Pandemic fear" and COVID-19: Mental health burden and strategies. Brazilian Journal of Psychiatry, 42(3), 232-235. https://doi.org/10.1590/1516-4446-2020-0008

Pakpour, A., \& Griffiths, M. (2020). The fear of COVID-19 and its role in preventive behaviors. Journal of Concurrent Disorders [revista en Internet] 2020 [acceso 9 de octubre de 2020]; 1(2020): 1-6. Journal of Concurrent Disorders, 2, 58-63.

Salmela-Aro, K., \& Read, S. (2017). Study engagement and burnout profiles among Finnish higher education students. Burnout Research, 7(August), 21-28. https://doi. org/10.1016/j.burn.2017.11.001

Schaufeli, W. B., \& Bakker, A. B. (2004). Job demands, job resources, and their relationship with burnout and engagement: A multi-sample study. Journal of Organizational Behavior, 25(3), 293-315. https://doi.org/10.1002/job.248

Schaufeli, W. B., Martínez, I. M., Pinto, A. M., Salanova, M., \& Barker, A. B. (2002). Burnout and engagement in university students a cross-national study. Journal of Cross-Cultural Psychology, 33(5), 464-481. https://doi.org/10.1177/0022022102033005003

Schnettler, T., Bobe, J., Scheunemann, A., Fries, S., \& Grunschel, C. (2020). Is it still worth it? Applying expectancy-value theory to investigate the intraindividual motivational process of forming intentions to drop out from university. Motivation and Emotion, 44(4), 491-507. https://doi.org/10.1007/s11031-020-09822-w

Seale, H., Leask, J. L., Po, K., \& MacIntyre, C. R. (2009). Will they just pack up and leave? Attitudes and intended behaviour of hospital health care workers during an influenza pandemic. BMC Health Services Research, 9(1), 1-8. https://doi.org/10.1186/14726963-9-30 
Seidel, G., \& Back, A. (2009). Success factor validation for global ERP programmes. 17th European Conference on Information Systems, ECIS 2009, 1-13.

Snell, W. E., \& Finney, P. D. (1998). Multidimensional AIDS Anxiety Questionnaire. In C. M. Davis, W. L. Yarber, R. Bauserman, G. Schreer, \& S. L. Davis (Eds.), Handbook of Sexuality-Related Measures. Routledge.

Tsamakis, K., Rizos, E., Manolis, A., Chaidou, S., Kympouropoulos, S., Spartalis, E., Spandidos, D., Tsiptsios, D., \& Triantafyllis, A. (2020). [Comment] COVID-19 pandemic and its impact on mental health of healthcare professionals. Experimental and Therapeutic Medicine, 19(6), 3451-3453. https://doi.org/10.3892/ etm.2020.8646

Van Lancker, W., \& Parolin, Z. (2020). COVID-19, school closures, and child poverty: a social crisis in the making. The Lancet Public Health, 5(5), e243-e244. https://doi. org/10.1016/S2468-2667(20)30084-0

Vanguardia. (2020, May 6). Universidades afirman que las inscripciones pueden disminuir un 50\% por COVID-19. Vanguardia. https://www.vanguardia.com/colombia/ universidades-afirman-que-las-inscripciones-pueden-disminuir-un-50-por-covid19-DC2325674

Wei, X., Wang, R., \& Macdonald, E. (2015). Exploring the relations between student cynicism and student burnout. Psychological Reports, 117(1), 103-115. https://doi. org/10.2466/14.11.PR0.117c14z6

Wong, A. P. (2020, May 5). Reconoce SEP colaboración de las universidades en lucha contra covid-19. Milenio. https:/www.milenio.com/politica/coronavirus-sepreconoce-apoyo-universidades-covid-19

Recibido: 26 de diciembre de 2020

Aceptado: 22 de febrero de 2021 